\title{
Introduction : Littératies et autonomie des apprenants avancés de langue(s)
}

\author{
Nikolay Slavkov \\ nikolay.slavkov@uottawa.ca
}

UNIVERSITÉ D'OTTAWA

Le présent volume compte dix articles portant sur les littératies et l'autonomie des apprenants de langue de niveau supérieur, thématique principale du colloque 2014 du CCERBAL organisé par l'ILOB et accueilli sur le campus de l'Université d'Ottawa. Cette thématique a été examinée sous optiques diverses et par différentes disciplines savantes durant le colloque, reflétant le travail lié aux principaux axes d'expertise de l'ILOB : l'enseignement et l'évaluation des langues, la politique linguistique, et l'apprentissage des langues assisté par la technologie. D'ailleurs, les articles de ce numéro s'articulent autour de ces axes fondamentaux et exposent une diversité de perspectives émanant de chercheurs renommés et d'universitaires émergents. Certains articles s'inspirent de projets de recherche achevés, d'autres rendent compte de travaux en cours ou d'études pilotes ou encore font état d'ateliers pédagogiques. Ce mélange d'approches et de perspectives dénote la nature dynamique du domaine et souligne la solide culture de recherche et d'enseignement encouragée par l'ILOB et son centre de recherche CCERBAL.

Un article principal sert d'introduction à ce volume, et le reste des articles est réparti en deux sections : Recherche et Perspectives pédagogiques. La première section renferme les articles fondés sur des études empiriques, alors que la seconde présente les articles inspirés de réflexions sur les méthodes didactiques et d'ateliers d'enseignement. Certains articles pourraient s'inscrire dans ces deux catégories et font ressortir l'interconnexion et, dans certains cas, la fusion de la recherche et de la pratique. Ce constat nous incite à marquer une pause pour réfléchir aux démarcations traditionnelles servant à conceptualiser les communications savantes.

\section{Article principal}

Ce numéro commence par l'article principal, «Le développement de l'autonomie dans l'apprentissage des langues et cultures étrangères : Le cas de l'Université de Strasbourg», par l'une des conférencières plénières du colloque, 
Nicole Poteaux. Universitaire renommée du domaine de l'autonomie des apprenants, Poteaux expose de riches expériences et exemples de son travail de longue date dans cette discipline en s'arrêtant au changement de paradigme des approches d'apprentissage linguistique centrées sur l'enseignant aux approches centrées sur l'apprenant ou autodidactes. Dans ce virage, les apprenants délaissent leur rôle passif traditionnel de destinataires de l'instruction pour se transformer en acteurs autonomes responsables, pouvant faire appel à un vaste éventail de ressources, de modalités et d'interlocuteurs. Par ailleurs, l'enseignant de langue acquiert de nouveaux rôles : auditeur, observateur attentif et conseiller, qui offre des suggestions non normatives et engage un dialogue pédagogique avec les apprenants.

\section{Recherche}

Le premier article de cette section, «Les compétences en français : Agentivité et motivation des étudiants universitaires », par Krystyna Baranowski, fait aussi écho au thème de l'autonomie. Baranowski présente les résultats préliminaires d'une série d'entrevues avec des étudiants francophones, anglophones et allophones d'une petite université franco-manitobaine. Pour remédier à la difficulté à maîtriser le français, signalée par des étudiants, Baranowski constate que l'intégration d'éléments du Portfolio canadien des langues pour enseignants (ACPLS/CASLT, 2010) favorise l'autonomisation et la responsabilité linguistique qui peuvent, en bout de ligne, procurer des avantages durables aux apprenants.

L'article suivant, «Engaging the hidden curriculum within the Canadian Language Benchmarks (2000 and 2012) as a complicated conversation», par Douglas Fleming, s'interroge sur la mesure dans laquelle un cadre d'évaluation peut être perçu comme distinct du programme d'études. L'auteur propose un examen des Niveaux de compétence linguistique canadiens (PawlikowskaSmith, 2000; Hajer et Kaskens, 2012) et soutient que les immigrants qui apprennent l'anglais peuvent être acculturés de façons spécifiques par l'instruction de la langue fondée sur ces niveaux de compétence. Il invite à adopter une perspective élargie de l'enseignement et de l'évaluation des langues qui intègre manifestement les valeurs de la citoyenneté et de l'équité.

Le troisième article de la section, «Developing autonomy through awareness of textual features in academic texts », par Hedy McGarrell, s'attarde aux textes et aux rapports universitaires sur des études empiriques auprès d'étudiants postsecondaires reposant sur le Corpus of Contemporary American English (COCA) (Davies, 2008) et le Compleat Lexical Tutor (Cobb, 2015). Après une analyse des rédactions effectuées par les étudiants et des entrevues de participants et d'enseignants, l'auteure a constaté que l'utilisation des outils des deux corpus représente un avantage pour la sensibilisation aux conventions 
lexicales et à la structure de la phrase.

L'article suivant, «Canadian English teachers : Volunteering to teach their mother tongue in a foreign country », par Gloria Romero, nous conduit au Chili et présente une recherche qualitative portant sur des bénévoles canadiens dans des écoles publiques locales. L'auteure applique des perspectives expérientielles et socioculturelles (Dewey, 1938; Vygotsky, 1978) et examine les thèmes ressortant des méthodes pédagogiques et des expériences personnelles des bénévoles dans leur nouvel environnement.

Cette section prend fin avec l'article, «La place de la compétence paraphrastique dans le Cadre européen commun de référence pour les langues », par Alexandra Tsedryk. La compétence paraphrastique est un aspect important de l'emploi d'une langue par les apprenants, non seulement à titre d'indicateur de la richesse lexicale et grammaticale, mais aussi de la communication efficace. L'auteure soutient que les apprenants pourraient bénéficier du rôle plus important accordé au concept de la reformulation, même s'il s'agit déjà d'un élément constitutif du CECRL (Conseil de l'Europe, 2001).

\section{Perspectives pédagogiques}

Le premier article de cette section, «Towards narrative-centred digital texts for advanced second language learners », par Nolan Bazinet, aborde l'enseignement de l'anglais langue seconde au Québec et l'utilisation de la technologie et des nouveaux médias en salle de classe. Bazinet met à contribution ses expériences d'enseignant de langue au niveau collégial et propose de nouvelles façons de motiver les étudiants et de retenir leur attention dans la concurrence avec les appareils électroniques (p. 86). L'auteur explore les jeux numériques narratifs comme outils pédagogiques et soutient qu'ils favorisent l'emploi par les apprenants de niveau supérieur d'une langue figurative et imbue de culture dans les milieux autonomes.

Stacia Johnson examine aussi l'utilisation de nouveaux médias et des technologies en classe dans son article, «Reaping the benefits of using Twitter in advanced language learning », qui tisse des liens entre le constructivisme social (Vygotsky, 1978) et le microblogage. L'auteure expose les pratiques didactiques dans un cours de français langue seconde du secondaire et discute les avantages potentiels découlant de la participation et de la collaboration des étudiants et leur sentiment général d'appartenance qui favorise leur apprentissage linguistique.

L'article, «Advanced English language acquisition and application in specialized subject areas », par Lucia Taylor, table sur le cadre sociologique pour suggérer une conception de l'enseignement de langue seconde ciblant des domaines spécialisés. Elle avance que les compétences générales en jeu dans l'assimilation d'une langue, dans le but d'assurer la communication efficace, sont 
importantes pour l'apprentissage du contenu spécialisé et l'acquisition d'un savoir-faire, de façon que les apprenants de l'anglais puissent s'intégrer pleinement au marché du travail canadien.

Le dernier article du numéro, «Pour que les étudiants de FLS comprennent et participent à la francophonie canadienne», par Laurence Thibault, MarieClaude Dansereau et Sylvie Lamoureux, approfondit le thème de la littératie socioculturelle et sociolinguistique. Il y est question de la nécessité pour les enseignants de français langue seconde de développer les littératies chez les communautés francophones du Canada. Prenant appui sur la recherche précédente, les auteures se penchent sur deux types de méthodes pédagogiques. La première concerne la compétence interculturelle, et la seconde vise une compréhension des variantes linguistiques locales et de leur statut symbolique.

\section{Références}

Association canadienne des professeurs de langue seconde/Canadian Association of Second Language Teachers (ACPLS/CASLT). 2010. Portfolio canadien des langues pour enseignants. Ottawa, ACPLS/CASLT.

Cobb, T. 2015. The Compleat Lexical Tutor, v. 8. Disponible à : www.lextutor.ca.

Conseil de l'Europe. 2001. Un cadre européen commun de référence pour les langues : apprendre, enseigner, évaluer. Paris, Didier. Disponible à : www.coe.int/t/dg4/linguistic/ Source/Framework_FR.pdf.

Davies, M. 2008-. The Corpus of Contemporary American English : 450 million words, 1990-present. Disponible à : corpus.byu.edu/coca/.

Dewey, J. 1938. Experience and education. New York : Collier.

Hajer, A. et A. Kaskens. 2012. Canadian language benchmarks 2012. Ottawa, Centre for Canadian Language Benchmarks.

Pawlikowska-Smith, G. 2000. Canadian language benchmarks 2000. Ottawa, Centre for Canadian Language Benchmarks.

Vygotsky, L.S. 1978. Mind in society : The development of higher psychological processes. Cambridge, MA, Harvard University Press. 